\title{
Discovery of a Novel Polyfluoroalkyl Benzenesulfonic Acid around Oilfields in Northern China
}

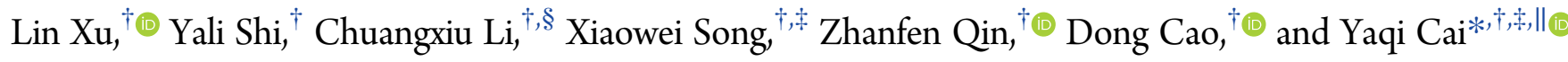 \\ ${ }^{\dagger}$ State Key Laboratory of Environmental Chemistry and Ecotoxicology, Research Center for Eco-Environmental Science, Chinese \\ Academy of Sciences, Beijing 100085, China \\ ${ }^{\ddagger}$ University of Chinese Academy of Sciences, Beijing 100049, China \\ ${ }^{\S}$ Institute of Environmental Science and Engineering, Qingdao University of Technology, Qingdao 266520, China \\ "Institute of Environment and Health, Jianghan University, Wuhan 430056, China
}

\section{Supporting Information}

ABSTRACT: The existence of more than 3000 per- and polyfluoroalkyl substances (PFASs) on the global market has prompted the identification and hazard characterization of hitherto unknown PFASs. In the present study, a novel PFAS, sodium $p$-perfluorous nonenoxybenzenesulfonate (OBS), was identified using Orbitrap MS/MS in water samples around a suspected application area, Daqing Oilfield, China. The peak OBS concentration was $3.2 \times 10^{3} \mathrm{ng} / \mathrm{L}$ in a sample taken near the oil well with the longest production history in Daqing. The concentrations of OBS and contribution to the sum of PFASs in surface waters displayed considerable variation among the three sampling areas (mean levels at $6.9,50$, and $5.6 \times 10^{2} \mathrm{ng} /$

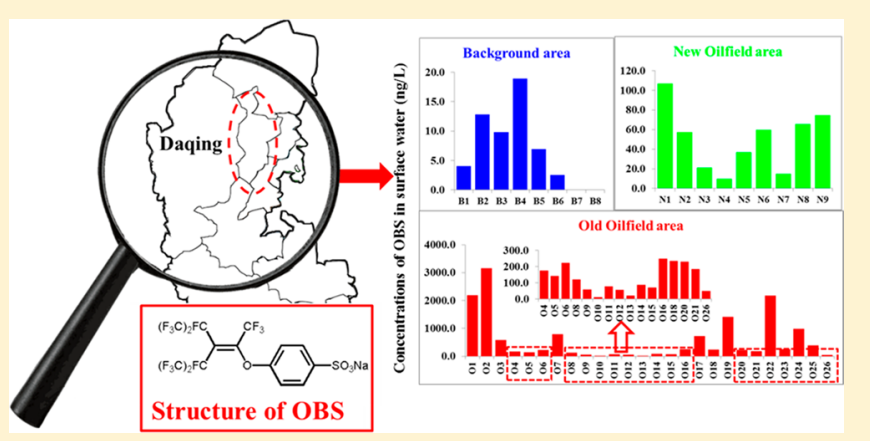
$\mathrm{L}$ with mean percentages at $9.8 \%, 45 \%$, and $69 \%$ in the background, new and old oilfield areas respectively) confirming that the density of oil wells and the oil production history are important factors influencing OBS contamination in the studied areas. A preliminary assessment of acute toxicity and environmental fate indicates that OBS exhibits similar toxicity and environmental persistence to perfluorooctanesulfonic acid (PFOS). The widespread occurrence of OBS, in conjunction with its potential hazard properties, underscores the need to further study on the bioaccumulation and potential for human exposure.

\section{INTRODUCTION}

Per- and polyfluoroalkyl substances (PFASs) are a group of synthetic chemicals that are widely used as surfactants and polymeric materials with numerous applications in industrial processes and consumer products. ${ }^{1}$ Owing to the high-energy $\mathrm{C}-\mathrm{F}$ bonds, perfluoroalkyl chains resist thermal and chemical degradation and are highly persistent in the environment. ${ }^{2}$ In addition to their persistence, long-chain perfluoralkyl sulfonic acids (PFSAs, $\mathrm{CF}_{2} \geq 6$ ) and perfluoroalkyl carboxylic acids (PFCAs, $\mathrm{CF}_{2} \geq 7$ ) are also bioaccumulative and toxic, ${ }^{3}$ which has triggered international regulation to restrict their production and usage. In 2009, perfluorooctanesulfonic acid (PFOS), its salts and perfluorooctanesulfonyl fluoride were listed as persistent organic pollutants (POPs) in Annex B under the Stockholm Convention. ${ }^{4}$ Perfluorooctanoic acid (PFOA) and its ammonium salt, as well as long-chain PFCAs, were added to the candidate list of Substances of Very High Concern (SVHCs) of EU REACH regulation in 2013. ${ }^{5}$ Since then, PFOA, its salts and PFOA-related compounds have been under consideration for inclusion in the list of POPs. ${ }^{6}$

In recent years, the decreasing concentrations of PFOS and PFOA in human blood suggest that the restrictions and voluntary elimination efforts have reduced human exposure in some developed countries. ${ }^{7-10}$ However, in addition to those well-known PFASs, approximately 3000 PFASs have been intentionally produced and used on the global market. ${ }^{11}$ These emerging PFASs include not only some alternative chemicals that have recently been introduced to replace long-chain PFSAs and PFCAs but also a large number of PFASs with a long history of production and use for different applications. ${ }^{12}$ The incomplete mass-balance of organic fluorine in environmental samples and human serum have also raised concerns about exposure to these hitherto unidentified PFASs. ${ }^{13,14}$

The development of high resolution mass spectrometry (HRMS) has provided scientists with powerful tools to identify novel organic compounds beyond a limited subset of known ones in complex samples. ${ }^{15}$ Taking advantage of the high mass accuracy and spectral resolving power, scientists have employed suspect-target or nontarget approaches to identify a large number of novel PFASs in the environment and industrial products. ${ }^{16,17}$ For example, a series of perfluoroether carboxylic

Received: August 23, 2017

Revised: November 14, 2017

Accepted: November 26, 2017

Published: December 8, 2017 


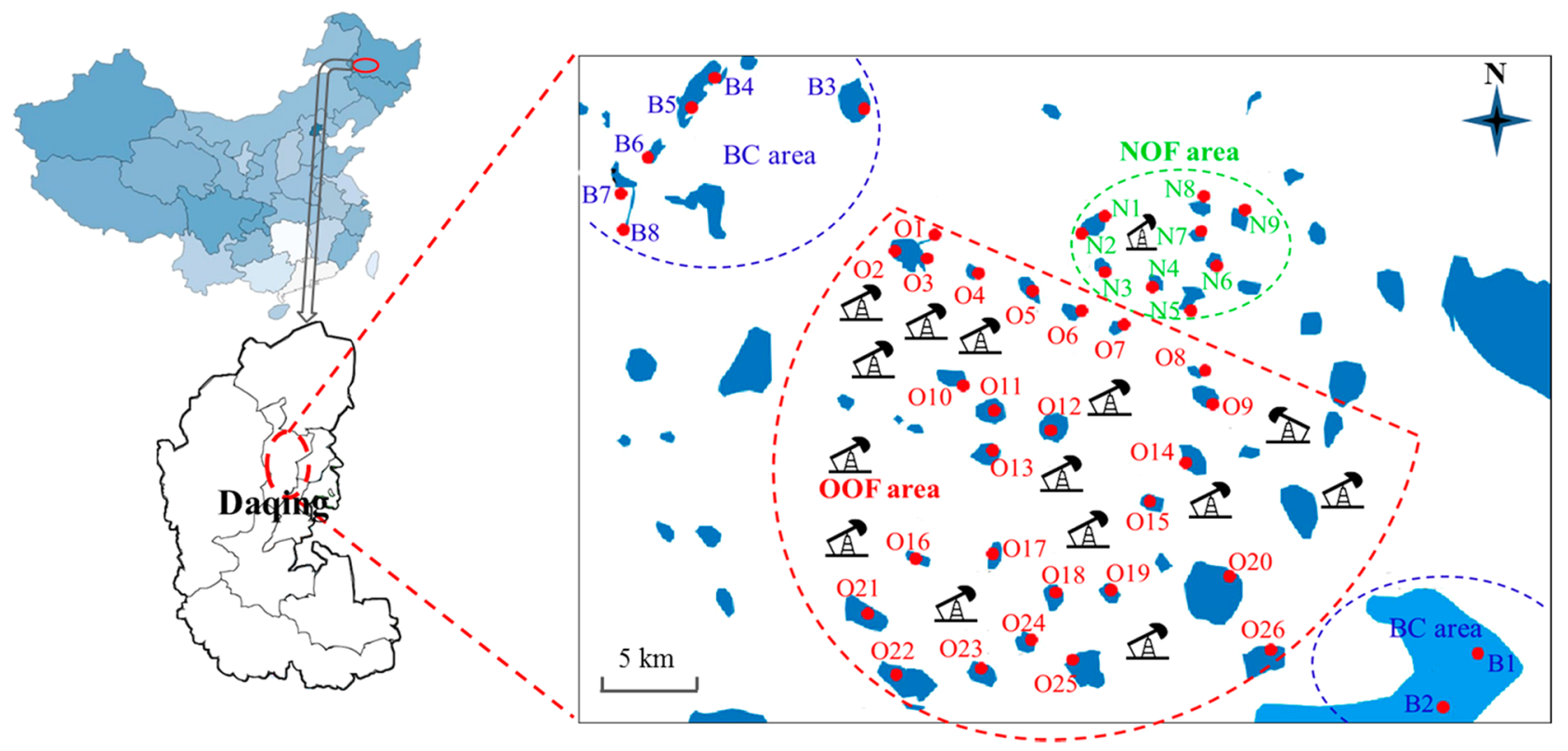

Figure 1. Sampling sites in Daqing Oilfield.

acids (PFECAs) and perfluoroether sulfonic acids (PFESAs) were identified in natural waters in North Carolina, using a nontargeted workflow with HR-TOFMS. ${ }^{16}$ Several polyfluorinated carboxylic acids, sulfonates and sulfates were observed in environmental samples from the Tennessee River near Decatur, Alabama. ${ }^{18}$ Numerous novel homologues and classes of PFASs were identified in aqueous film-forming foams (AFFFs), commercial surfactant concentrates or the surrounding impacted environment in the U.S. ${ }^{19-21}$ Gebbink et al. identified 11 emerging PFASs using a suspect-target screening analysis in the river and drinking water samples near a fluorochemical production plant. ${ }^{17} \mathrm{Liu}$ et al. discovered and confirmed the existence of three new classes of PFASs, including polyfluorinated sulfates, chlorine or hydro substituted perfluorocarboxylates, in industrial wastewater from China. ${ }^{22}$ Among the numerous novel PFASs recently being identified in the environment, some have been shown to significantly contribute to the fraction of organic fluorine in biota and humans. ${ }^{14,23,24}$ Even so, there are still many PFASs on the global market with little to no information on their occurrence, exposure and toxic effect in the environment. ${ }^{12}$

Through a detailed patent search we came across an informal report of a PFAS with a benzene ring moiety which has not been reported in the environment previously, named sodium $\mathrm{p}$ perfluorous nonenoxybenzenesulfonate (OBS). OBS related products, with the trade name Neos Ftergent (NF), have been produced by a Japanese company (Neos) since $1980 \mathrm{~s}^{25}$ The OBS was produced at a volume of 3500 tones per year in China $^{26}$ and has been widely used as an additive in film-forming fluoroprotein foams (FFFPs) and alcohol-resistant foams (AFFF-AR/FFFP-AR) ${ }^{27}$ and as a surfactant for improving oil production. $^{28}$ Unlike PFCAs and PFSAs which have been synthesized by electrochemical fluorination and/or telomerization, OBS is produced by oligomerization, in which hexafluoropropylene trimer (HFPT) is an important starting material. ${ }^{29-31}$ The synthesis process was shown in SI Figure S1. Although four isomers for HFPT oligomer (HFPT-a', $b^{\prime}, c^{\prime}$, and $\mathrm{d}^{\prime}$ shown in SI Figure S2) have been reported, HFPT- $\mathrm{c}^{\prime}$ exhibits much higher reactivity than the other three isomers. ${ }^{29}$
As a result, OBS with the structure c (OBS-c, SI Figure S2) was reported as the main product. ${ }^{30,31}$ To date, the studies on OBS have focused on investigating its physicochemical properties and optimizing the surface tension by mixing with different nonfluorinated surfactans. ${ }^{32,33}$ To the best of our knowledge, there is only one study reporting that OBS displays similar PBT properties with PFOS although OBS can be decomposed in UV and $\mathrm{UV} / \mathrm{H}_{2} \mathrm{O}_{2}$ system. $^{26}$ Regarding its occurrence, environmental behavior and fate, there is no available data.

The application of OBS as a surfactant in oilfield of China appears to be increasing with more oilfields entering the final stage, in which chemicals were used for improving oil recovery. PFOS has also been used for this purpose in oilfields in some parts of the world. ${ }^{11}$ In an informal report, which was the only report of this nature, the amount of PFOS released to the sea by Norwegian oil rigs between 1980 and 2005 was assessed to be more than 80 tons. $^{34}$ This indicates that contamination of both emerging and legacy PFASs is likely to occur around oilfields. Except for direct usage as additive agents in oil production, PFAS contamination around oilfields may also originate from the use in firefighting foam extinguishing agents for hydrocarbon-fueled fires. ${ }^{35}$ Therefore, we hypothesized that OBS and legacy PFASs would be widespread in its potential use area, oilfield in China. However, until now, very little data has been reported for PFASs in the petroleum industry, specifically, OBS has never been identified or reported in the environment.

In the present study, we employed Orbitrap HR-MS to confirm the molecular formula of OBS based on the measured accurate mass and major fragmentation ions and to identify its presence in water samples from areas surrounding an oilfield. Subsequently, the spatial distribution of OBS was investigated in areas surrounding Daqing Oilfield (the largest oilfield in China). For comparison, legacy PFASs, including PFCAs and PFSAs, were also measured and discussed with respect to their contrasting sources, transport and fate. Furthermore, acute toxicity tests were conducted for fish and frog tadpole in order to get 96h-LC50 of OBS and a screening-level tool, EPI suite (1.4.7), was used to estimate its degradation potential in the environment. 


\section{EXPERIMENTAL SECTION}

Chemicals and Reagents. Both a native standard solution PFAC-MXB, containing C4-C14 PFCAs and C4, C6, and C8 PFSAs, and a mass-labeled standard solution MPFAC-MXA, including ${ }^{13} \mathrm{C}_{4}-\mathrm{PFBA},{ }^{13} \mathrm{C}_{4}-\mathrm{PFOA},{ }^{13} \mathrm{C}_{2}-\mathrm{PFDoDA}$, and ${ }^{13} \mathrm{C}_{4}$-PFOS, were purchased from Wellington Laboratories (Guelph, ON, Canada). An authentic OBS standard ( ${ }^{19} \mathrm{~F}-\mathrm{NMR}$ results shown in SI Figure S3) was purified from the commercial product purchased from Kaierfu New Material Co., Ltd. (95\%, industrial grade) using an Autopurification HPLC system (Waters 2767, Milford, MA), with the separation on an Xbridge Prep C18 column $(19 \mathrm{~mm} \times 250 \mathrm{~mm}, 5 \mu \mathrm{m})$. Detailed information on the purification process is provided in the SI. HPLC-grade methanol was obtained from Fisher Scientific (Pittsburgh, PA). Ammonium acetate $\left(\mathrm{NH}_{4} \mathrm{OAc}\right.$, > 97\%), formic acid (HPLC grade) and ammonium hydroxide (v/v, 50\%, HPLC grade) were obtained from Alfa Aesar (Ward Hill, MA). Glass-fiber filter membranes $(0.7 \mu \mathrm{m}, 47 \mathrm{~mm})$ were purchased from Sartorius Stedim Biotech (Gottingen, Germany), and Oasis WAX solid-phase extraction cartridges (150 mg, 6 cc) from Waters Co. (Milford, MA). A Milli-Q Advantage A10 system (Millipore) was used to prepare ultrapure water $\left(>18.2 \mathrm{M} \Omega \mathrm{cm}^{-1}\right)$ used in the present study.

Sample Collection and Pretreatment. A total of 86 surface water samples were collected from groups of lakes in the Daqing Oilfield area, Heilongjiang Province, China, during two sampling campaigns (May and November 2015). The sampling sites $(n=43)$ are indicated in Figure 1. Eight samples (B1-B8) were collected from a background (BC) area located at about $15 \mathrm{~km}$ away from the dense oilfield area. Nine samples (N1-N9) were collected from a sparse and newly developed oilfield area with only a few oil-producing wells, hereafter referred to as the new oilfield (NOF) area. The remaining 26 samples (O1-O26) were collected from an old oilfield (OOF) area with a longer oil production history and very dense network of oil producing wells. 1L polypropylene (PP) bottles were used for sample collection after being rinsed with high purity water followed by methanol. During the sampling campaigns, bottles were submerged to approximately $0.4 \mathrm{~m}$ below the surface of lake, filled and then capped under water. All samples were transferred to the laboratory and pretreated using method described in the following section within 1 week.

The methods for extraction and cleanup were similar to our previously described methods for PFOS and other anionic PFASs. ${ }^{36,37}$ In brief, after being spiked with $2 \mathrm{ng}$ of an internal standard (MPFAC-MXA: ${ }^{13} \mathrm{C}_{4}-\mathrm{PFBA},{ }^{13} \mathrm{C}_{4}-\mathrm{PFOA},{ }^{13} \mathrm{C}_{4}-$ PFDoDA, and ${ }^{13} \mathrm{C}_{4}-$ PFOS), $200 \mathrm{~mL}$ of water was filtered through a $0.7 \mu \mathrm{m}$ glass fiber filter membrane. The filtering was followed by washing the filter with $5 \mathrm{~mL}$ methanol to avoid the loss of PFASs and the combined water and methanol was loaded onto a single-use cartridge (Oasis WAX, $6 \mathrm{cc} / 150 \mathrm{mg}$ ) that had been preconditioned using $4 \mathrm{~mL}$ of $0.1 \%$ ammonium hydroxide (in methanol), $4 \mathrm{~mL}$ of methanol and $4 \mathrm{~mL}$ of ultrapure water, sequentially, at a rate of $1 \mathrm{drop} / \mathrm{s}$. After sample loading, the cartridges were subsequently washed with $4 \mathrm{~mL}$ of buffer solution $(25 \mathrm{mmol} / \mathrm{L}$ acetic acid/ammonium acetate, $\mathrm{pH}$ 4) and $8 \mathrm{~mL}$ of ultrapure water. Then, the cartridges were centrifuged for $10 \mathrm{~min}$ at $3000 \mathrm{rpm}$ to remove the residual water. Finally, $4 \mathrm{~mL}$ of methanol and $4 \mathrm{~mL}$ of $0.1 \%$ ammonium hydroxide in methanol were used to elute the target compounds. The combined eluate was concentrated to $1 \mathrm{~mL}$ under gentle nitrogen gas flow prior to transferring to an injection vial.

Instrumental Analysis. OBS in surface water extracts was identified using HPLC with a dual pump, autosampler and column compartment (Ultimate 3000, Thermo Fisher Scientific) coupled with an Orbitrap Fusion MS system (Thermo Fisher Scientific, Waltham, MA). Chromatographic separation for identification was achieved using an Acclaim 120 C18 column $(5 \mu \mathrm{m}, 4.6 \mathrm{~mm} \times 150 \mathrm{~mm})$ with an injection volume of $10 \mu \mathrm{L}$, and the mobile phase was a mixture of methanol (eluent A) and $50 \mathrm{mM}$ ammonium acetate in water (eluent B) with a flow rate of $1.0 \mathrm{~mL} / \mathrm{min}$. The gradient program was as follows: The composition of eluent $\mathrm{B}$ decreased from $28 \%$ to $5 \%$ within $4 \mathrm{~min}$, and eluent B was held at $5 \%$ for $3 \mathrm{~min}$, increased to $28 \%$ within $0.1 \mathrm{~min}$, and held at $28 \%$ until $10 \mathrm{~min}$ to re-equilibrate the column. The Orbitrap MS was operated in negative ESI mode. Data were acquired using Xcalibur software (Thermo Fisher) in full scan mode (50-700 $\mathrm{m} / z$ ) with a resolution of 120000 . The capillary voltage was set at $2500 \mathrm{~V}$, and the ion transfer tube and vaporizer temperatures were 350 and $200{ }^{\circ} \mathrm{C}$, respectively. The sheath, aux, and sweep gas flow rates were set at 35,10 , and 1 Arb. The higher-energy collision dissociation (HCD) energy was $43 \%$.

OBS and other PFASs were quantified using HPLC (Ultimate 3000, Thermo Fisher Scientific) coupled with electrospray ionization (ESI) MS/MS (API 4500, Applied Biosystems/MDS SCIEX). The separation of OBS and other PFASs was conducted using an ACQUITY HSS PFP column $(1.8 \mu \mathrm{m}, 100 \AA$, $2.1 \mathrm{~mm} \times 50 \mathrm{~mm}$, Waters $)$ with an injection volume of $10 \mu \mathrm{L}$. The total analysis time was $28 \mathrm{~min}$, and the flow rate was $0.25 \mathrm{~mL} / \mathrm{min}$. The mobile phase consisted of methanol (eluent A) and $20 \mathrm{mM}$ ammonium hydroxide/20 $\mathrm{mM}$ formic acid in water $(\mathrm{pH} \mathrm{4}$, eluent B). The solvent gradient started at $90 \% \mathrm{~B}$, which was held for $3 \mathrm{~min}$; was ramped in $2.0 \mathrm{~min}$ to $50 \% \mathrm{~B}$; was decreased to $22 \% \mathrm{~B}$ by 20.0 $\mathrm{min}$ and to $0 \% \mathrm{~B}$ by $20.1 \mathrm{~min}$, where it was held for $2 \mathrm{~min}$; and was returned to the initial conditions by $22.1 \mathrm{~min}$, where it was held until to $28.0 \mathrm{~min}$. The ESI-MS/MS was operated in negative ESI mode and data were acquired using Analyst software (Applied Biosystems/MDS SCIEX). Related parameters and detailed MS transitions for OBS and legacy PFASs are shown in SI Table S1.

Quality Assurance, Whole Method Performance, and Statistical Analysis. Before the experiments were carried out, all solvents and laboratory consumables were checked for PFASs contamination, and one procedural blank was pretreated for each batch of samples $(n=10)$. In addition, one field blank, poly propylene bottle filled with Milli-Q water, was carried to the field site for each sampling campaign. However, the levels of PFASs in those blanks and solvents were consistently lower than the instrumental detection limits. A new calibration curve was constructed if there was a deviation higher than $\pm 20 \%$ from the initial level of calibration standard, which was injected after every batch of samples $(n=10)$.

To evaluate the accuracy and precision of the whole method, spiked-recovery tests were carried out using surface water samples with nondetectable levels of OBS and low concentration of legacy PFASs. Briefly, 2 ng of OBS and PFAC-MXB was added to surface water samples $(n=5)$ prior to adding $2 \mathrm{ng}$ of MPFAC-MXA as internal standard. An additional set of surface water samples were spiked only with $2 \mathrm{ng}$ of MPFACMXA. The water samples were subsequently treated according to the procedure described above. The extraction efficiencies 

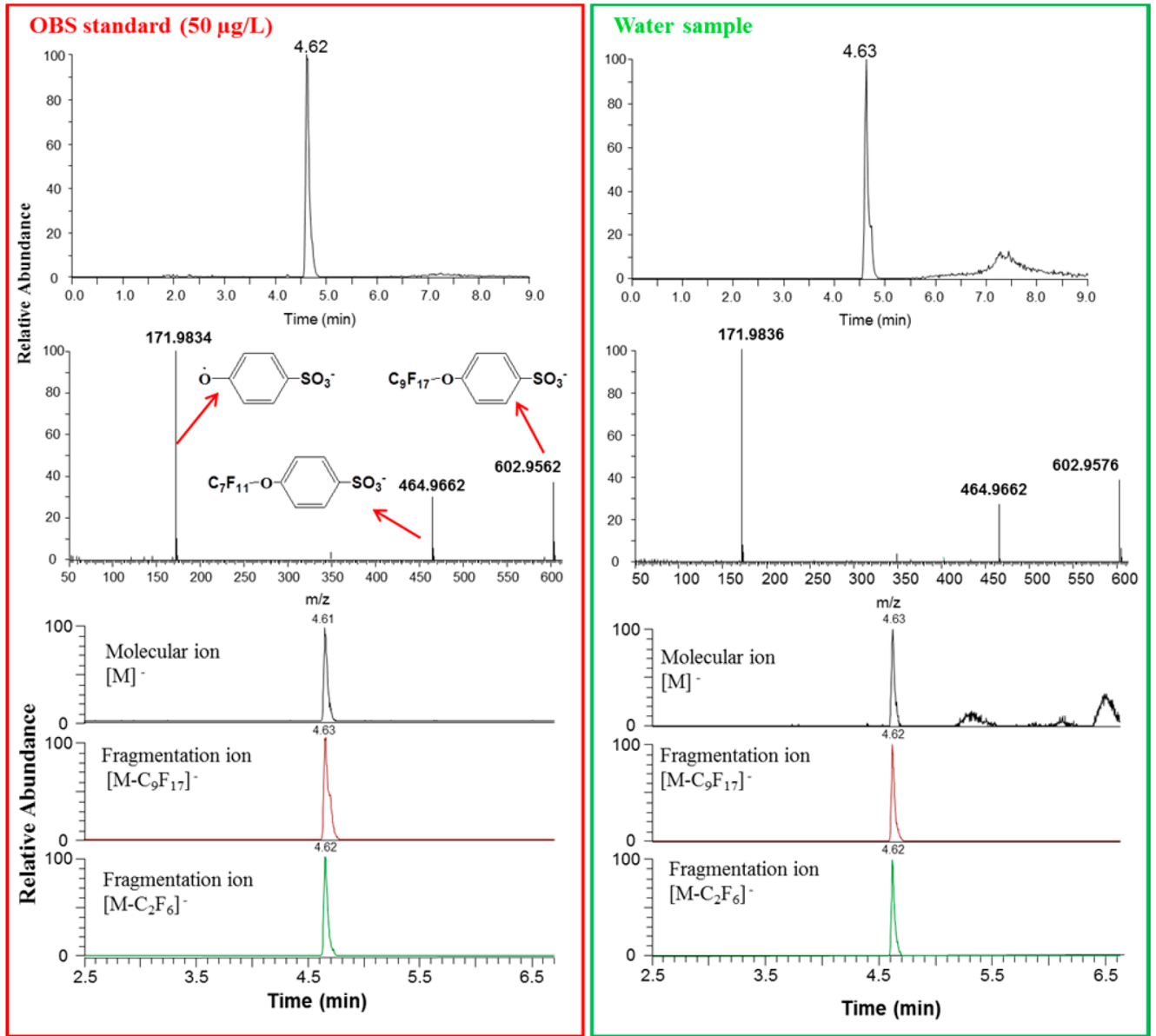

Figure 2. Chromatogram and mass spectra of the characteristic MS2 fragmentation ions for an OBS standard and a real sample. Left: OBS standard solution $(50 \mathrm{ng} / \mathrm{mL})$, Right: water extract sample.

(EEs) for OBS, native and isotopically labeled PFASs were calculated using the following equation:

$$
\mathrm{EE}(\%)=\frac{\left(A_{\mathrm{sp}}-A_{\mathrm{nsp}}\right)}{A_{\mathrm{st}}} \times 100
$$

where $A_{\text {sp }}$ and $A_{\text {nsp }}$ referred to the peak areas resulting from the extracted spiked and nonspiked samples, respectively. $A_{s t}$ is the area from a standard solution in methanol at the same spiking level $(2 \mathrm{ng} / \mathrm{mL})$. The results showed that EEs for OBS, native and isotopically labeled PFASs were in the acceptable range (70 \pm 4.1 to $120 \pm 5.2 \%$ ), with the relative standard deviations (RSDs) lower than $8.4 \%$ (detail information shown in SI Table S2). The acceptable EEs indicated that there are no significant matrix effects for all target compounds.

The matrix recoveries (MRs) for OBS and legacy PFASs referred to relative recoveries with internal standard correction. The equation for calculating MRs is as follows:

$$
\operatorname{MR}(\%)=\frac{\left(C_{\mathrm{msp}}-C_{\mathrm{mnsp}}\right)}{C_{\mathrm{sp}}} \times 100
$$

Here, $C_{\mathrm{msp}}$ and $C_{\mathrm{mnsp}}$ referred to the measured concentrations of spiked and nonspiked samples, whereas $C_{\text {sp }}$ is the spiked concentration $(2 \mathrm{ng} / \mathrm{mL})$. The mean $\mathrm{MRs}$ of the PFASs, including OBS, ranged from $80 \pm 9.1$ to $110 \pm 5.5 \%$, demonstrating that OBS can be confidently quantified in surface water using similar methodologies to those of legacy PFASs. A series of standard solution in methanol $(0.05,0.1,0.5$,
$1.0,5,10,20$, and $50 \mathrm{ng} / \mathrm{mL}$ ) were used to establish 8-point calibration curve for quantification using internal standard approach, showing strong linearity $\left(1 / \mathrm{x}^{2}\right.$ weighted) with correlation coefficients $\left(r^{2}\right)$ higher than 0.99 . Meanwhile, the method limits of detections (MLDs) were calculated using the equation:

$$
\mathrm{MLD}=\frac{(\sigma \times 3.3-b)}{a}
$$

Here, $a$ and $b$ is the slope and y-intercept of the calibration curve; $\sigma$ is $1 / x^{2}$-weighted regression-residual standard deviation. The method limits of quantifications (MLQs, $0.05 \mathrm{ng} / \mathrm{L}$ to $0.96 \mathrm{ng} / \mathrm{L}$, details shown in SI Table S2) were calculated by multiplying MLD with 3.

IBM PASW statistics 18.0 (SPSS Inc., 1993-2007) software was used to perform statistical analysis. The differences of concentrations among three studied areas were evaluated through nonparametric Mann-Whitney U-test. The relationships between OBS and PFOS or other PFASs concentrations were tested with Spearman's rank correlation analysis. All results are based on a statistical significance threshold of $p<$ 0.05 .

Fish and Frog Tadpole Acute Toxicity Test. Zebrafish (Danio rerio) (2.5 months old, $2.5 \mathrm{~cm}$ ), purchased from the Institute of Hydrobiology, Chinese Academy of Science (Wuhan, China), were acclimatized for 7 days prior to testing and fed three times a day until $24 \mathrm{~h}$ before the test began. The water quality parameters were as follows: chlorine concen- 
aBS $\square$ PFCAs $\square$ PFSAs

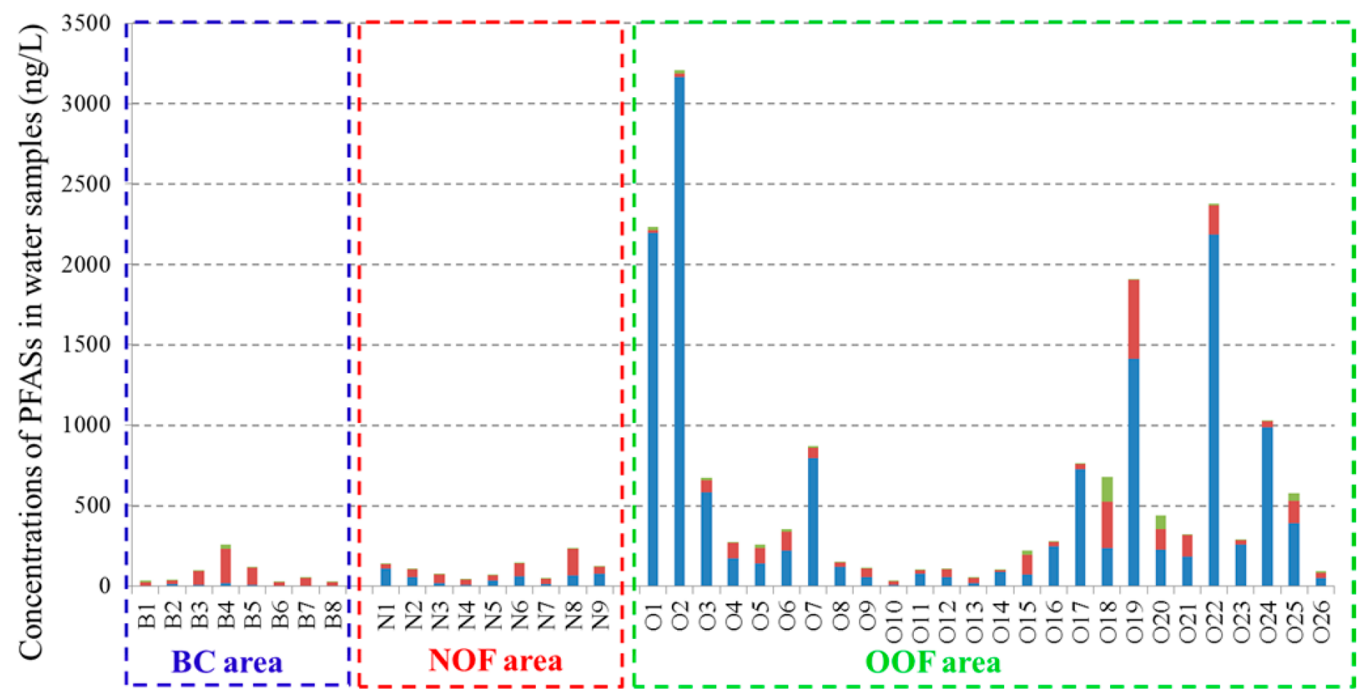

Figure 3. Stacked plots of OBS, PFCAs, and PFSAs concentrations in individual water samples from three studied areas.

tration of $<5 \mu \mathrm{g} / \mathrm{L}, \mathrm{pH}$ 6.5-7.0, dissolved oxygen concentration of $>5 \mathrm{mg} / \mathrm{L}$, and water hardness $\left(\mathrm{CaCO}_{3}\right)$ of approximately $150 \mathrm{mg} / \mathrm{L}$. The water temperature was maintained at $23 \pm 1{ }^{\circ} \mathrm{C}$. The photoperiod was $12: 12 \mathrm{~h}$ light:dark. The fish acute toxicity test was conducted following the Organization for Economic Cooperation and Development (OECD) Guideline 203. ${ }^{38}$ The water was aerated for $24 \mathrm{~h}$ prior to the start of the test. Ten fish were placed in $3 \mathrm{~L}$ of test water, and the water was renewed every $24 \mathrm{~h}$. Six nominal OBS concentrations $(0,11.3,14.5,18.9,24.6$, and $32 \mathrm{mg} / \mathrm{L})$ were used with each treatment in triplicate. According to OECD $203,^{38}$ the geometric mean of the highest concentration causing no mortality and the lowest concentration producing $100 \%$ mortality was calculated as the LC50 because the data obtained were inadequate for the use of standard methods for calculating the LC50.

The acute toxicity of OBS was also tested using the BlackSpotted frogs (Pelophylax nigromaculatus), according to a previous method ${ }^{39}$ with minor adjustments. Adult frogs were the offspring of frogs from a P. nigromaculatus farm in Bengbu, Anhui Province, China. P. nigromaculatus was raised as described in the previous study. ${ }^{40}$ In brief, adult $P$. nigromaculatus frogs were housed in an amphibious aquarium containing sand and dechlorinated water. The water quality for raising $P$. nigromaculatus was the same as that for the zebrafish. Two pairs of adult frogs were induced to breed by injection of $20 \mu \mathrm{g}$ of luteinizing hormone-releasing hormone (Ningbo Second Hormone Factory, China) and human chorionic gonadotropin (Yantai North Pharmaceutical Co. Ltd., China; $300 \mathrm{IU}$ for female, $200 \mathrm{IU}$ for male) dissolved in $0.6 \% \mathrm{NaCl}$, accompanied by simulated rainfall. Fertilized eggs were incubated in dechlorinated tap water. After reaching stage $27,{ }^{41} 10$ tadpoles were placed in a tank containing $400 \mathrm{~mL}$ of test water to ensure a loading capacity of less than $1 \mathrm{~g} / \mathrm{L}$. Six nominal OBS concentrations $(0,13.7,19.2,26.9,37.6$, and 52.7 $\mathrm{mg} / \mathrm{L}$ ) were used with each treatment in triplicate. Similar to the fish acute toxicity test, the geometric mean of the highest concentration causing no mortality and the lowest concentration producing $100 \%$ mortality was calculated as LC50.

\section{RESULTS AND DISCUSSION}

Identification and Quantification of OBS. A reference standard of OBS was obtained by purifying a commercial OBS product and its structure was confirmed via fluorine nuclear magnetic resonance $\left({ }^{19} \mathrm{~F}-\mathrm{NMR}\right.$, SI Figure S3). First, the reference standard and sample extract solution was run in LC-Orbitrap MS system with full-scan mode. As shown in Figure 2, the accurate mass of OBS in the standard solution and sample extract was 602.9562 and 602.9576 with a mass error $(\Delta \mathrm{m})$ of 1.8 and $3.7 \mathrm{ppm}$ when designating the same elemental composition as OBS. Furthermore, a signal (604.9514 and $604.9582)$ at $m / z+2 \mathrm{Da}$ with a relative intensity of approximately $4.5 \%$ was observed for an isotope of sulfur $\left({ }^{34} \mathrm{~S}\right)$, indicating the presence of one sulfur atom in the molecule. Second, a data-dependent $\mathrm{MS}^{2}$ (dd-MS ${ }^{2}$ ) scan was performed using $\mathrm{m} / z=602.96$ as parent ion to facilitate the structural elucidation of OBS. In $\mathrm{MS}^{2}$ spectrum (Figure 2), two main fragments were observed with $\mathrm{m} / z$ at 464.9662 and 171.8834 for the reference standard solution. The fragment with $m / z=464.9662\left(\left[\mathrm{C}_{7} \mathrm{~F}_{11} \mathrm{C}_{4} \mathrm{H}_{4} \mathrm{SO}_{4}\right]^{-}\right)$was produced by a loss of $-\mathrm{C}_{2} \mathrm{~F}_{6}$, and the other fragment $(\mathrm{m} / z=171.8834$, $\left[\mathrm{C}_{4} \mathrm{H}_{4} \mathrm{SO}_{4}\right]^{-}$) corresponded to $\left[\mathrm{M}-\mathrm{C}_{9} \mathrm{~F}_{17}\right]^{-}$, as shown in Figure 2. The mass errors for two main fragments were 2.1 and 4.7 ppm when designating the same elemental composition as $\left[\mathrm{C}_{11} \mathrm{~F}_{11} \mathrm{H}_{4} \mathrm{SO}_{4}\right]^{-}$and $\left[\mathrm{C}_{4} \mathrm{H}_{4} \mathrm{SO}_{4}\right]^{-}$, respectively. The $\mathrm{MS}^{2}$ spectrum of water sample extract displayed identical major fragmentation ions, $[\mathrm{M}]^{-},\left[\mathrm{M}-\mathrm{C}_{2} \mathrm{~F}_{6}\right]^{-}$, and $\left[\mathrm{M}-\mathrm{C}_{9} \mathrm{~F}_{17}\right]^{-}$, demonstrating that these fragmentation ions originated from OBS. In addition, retention times of the major fragments ([M$\left.\mathrm{C}_{2} \mathrm{~F}_{6}\right]^{-}$and $\left.\left[\mathrm{M}-\mathrm{C}_{9} \mathrm{~F}_{17}\right]^{-}\right)$produced by the OBS standard (4.61-4.63 $\mathrm{min})$ and the real water samples (4.62-4.63 $\mathrm{min}$ ) displayed excellent agreement which further supports the presence of OBS in the surface water. Given the availability of a reference standard, which allowed confirmation based on retention time, accurate mass and two product ions, OBS could be identified in water samples according to the highest confidence level proposed by Schymanski et al. ${ }^{42}$

For quantification of OBS and legacy PFAS, a chromatographic method using a PFP column was chosen due to its improved peak separation and reduction of matrix suppression/ enhancement effects. Under these conditions, two or three 
Table 1. Statistics Analysis for the Difference of PFASs Concentration among Three Areas (Mann-Whitney Test)

\begin{tabular}{|c|c|c|c|c|c|c|c|c|c|c|}
\hline \multicolumn{11}{|c|}{ Mann-Whitney Test } \\
\hline group & & OBS & PFOS & PFBS & PFBA & PFPeA & PFHxA & PFHpA & PFOA & PFNA \\
\hline \multirow[t]{5}{*}{$\mathrm{BC}$ and $\mathrm{NOF}$ area } & Mann-Whitney U & 3.00 & 29.00 & 7.00 & 31.00 & 33.00 & 30.00 & 35.00 & 32.00 & 14.00 \\
\hline & Milcoxon W & 39.00 & 65.00 & 52.00 & 67.00 & 69.00 & 66.00 & 80.00 & 68.00 & 50.00 \\
\hline & $\mathrm{Z}$ & -3.18 & -0.67 & -2.83 & -0.48 & -0.29 & -0.58 & -0.10 & -0.38 & -2.12 \\
\hline & Asymp. Sig. (2-tailed) & 0.00 & 0.50 & 0.00 & 0.63 & 0.77 & 0.56 & 0.92 & 0.70 & 0.03 \\
\hline & Exact Sig. $\left[2^{*}(1 \text {-tailed Sig. })\right]^{a}$ & 0.00 & 0.54 & 0.00 & 0.67 & 0.81 & 0.61 & 0.96 & 0.74 & 0.04 \\
\hline \multirow[t]{5}{*}{$\mathrm{BC}$ and $\mathrm{OOF}$ area } & Mann-Whitney U & 2.00 & 42.00 & 98.50 & 99.00 & 93.00 & 89.00 & 93.00 & 77.00 & 61.00 \\
\hline & Milcoxon W & 38.00 & 78.00 & 449.50 & 135.00 & 129.00 & 125.00 & 129.00 & 113.00 & 97.00 \\
\hline & $\mathrm{Z}$ & -4.14 & -2.52 & -0.22 & -0.20 & -0.45 & -0.61 & -0.45 & -1.10 & -1.75 \\
\hline & Asymp. Sig. (2-tailed) & 0.00 & 0.01 & 0.82 & 0.84 & 0.66 & 0.54 & 0.66 & 0.27 & 0.08 \\
\hline & Exact Sig. $\left[2^{*}(1 \text {-tailed Sig. })\right]^{a}$ & 0.00 & 0.01 & 0.83 & 0.86 & 0.68 & 0.56 & 0.68 & 0.29 & 0.08 \\
\hline \multirow[t]{5}{*}{ NOF and OOF area } & Mann-Whitney U & 33.00 & 20.00 & 53.00 & 108.00 & 101.00 & 110.00 & 99.00 & 78.00 & 105.00 \\
\hline & Milcoxon W & 78.00 & 65.00 & 98.00 & 459.00 & 146.00 & 155.00 & 144.00 & 123.00 & 150.00 \\
\hline & $\mathrm{Z}$ & -3.17 & -3.66 & -2.45 & -0.34 & -0.60 & -0.26 & -0.68 & -1.47 & -0.45 \\
\hline & Asymp. Sig. (2-tailed) & 0.00 & 0.00 & 0.01 & 0.73 & 0.55 & 0.79 & 0.50 & 0.14 & 0.65 \\
\hline & Exact Sig. $\left[2^{*}(1 \text {-tailed Sig. })\right]^{a}$ & 0.00 & 0.00 & 0.01 & 0.75 & 0.57 & 0.81 & 0.52 & 0.15 & 0.67 \\
\hline
\end{tabular}

peaks were observed for the major OBS transition (603.2/ 172.0) of the OBS commercial product solution and the samples with the highest concentrations (SI Figure S4). For the second transition 603.1/464.9 only one main peak at the retention time of 14.29 was observed. As mentioned above, the fragment of 464.9 is the product ion of OBS with the loss of $-\mathrm{C}_{2} \mathrm{~F}_{6}$. Thus, the phenomenon that only one peak was observed for 603.1/464.9 may be because it is more difficult to lose this fragment for isomer OBS-a, OBS-b, and OBS-d. Therefore, although the OBS-c, which was confirmed using ${ }^{19} \mathrm{~F}-\mathrm{NMR}$ in the present study, was the main product due to higher activity of HFPT- $\mathrm{c}^{\prime}$, the existence of the other isomers is also possible. Authentic and mass-labeled standards of individual OBS isomers would be needed to enhance the analytical accuracy and study the environmental behavior and fate. However, in the present study, we only reported the concentrations of OBS-c because of the low detection frequency of the other isomers.

Occurrence of OBS and Other PFASs in the Surrounding Environment of the Oilfield. Among the 15 analytes included in this study, OBS, PFOS, PFBS, and C4-C9 PFCAs were detectable in surface water (SI Table S3). OBS was consistently found at the highest concentrations ranging from < MLQ to $3.2 \times 10^{3} \mathrm{ng} / \mathrm{L}$ with the detection frequency (DF) at 95\%. Relatively low levels were observed for PFBS and PFOS in the range of $<$ MLQ-19 and $<$ MLQ-1.5 $\times 10^{2}$, with the DF at $72 \%$ and $98 \%$. Among the detectable PFCAs, PFBA was the major contributor, accounting for $36-55 \%$, 39-53\%, and $18-57 \%$ of the total PFCA concentration ( $\sum$ PFCAs) in the $\mathrm{BC}, \mathrm{NOF}$, and $\mathrm{OOF}$ areas, respectively. The concentrations of PFBA ranged from 3.0 to $2.4 \times 10^{2} \mathrm{ng} / \mathrm{L}$ in three areas. The other detectable PFCAs, PFPeA, PFHxA, PFHpA, and PFOA, contributed similarly to $\sum$ PFCAs, with mean (median) percentages in the range of $9.6-18 \%(8.5-18 \%)$. The concentrations of PFPeA, PFHxA, PFHpA, PFOA, and PFNA were in the range of $0.67-1.8 \times 10^{2}, 1.4-42,1.9-23,2.2-46$, and $0.58-11 \mathrm{ng} / \mathrm{L}$, respectively.

As illustrated in Figure 3, OBS contributed the most to the total PFAS concentrations ( $\sum$ PFASs $=$ OBS $+\sum$ PFCAs + $\sum$ PFSAs) in the samples from the NOF and OOF areas, where the proportions of OBS were in the range of $24-79 \%$ (mean:
45\%; median: 41\%) and 32-99\% (mean: 69\%; median: 70\%), respectively. However, for samples in the BC area, OBS only accounted for $0-33 \%$ (mean: 9.8\%; median: $8.9 \%$ ) of $\sum$ PFASs. The OBS levels also differed greatly between the three sampling areas. The mean (median) OBS concentrations in the NOF and OOF areas, $50(57)$ and $5.6 \times 10^{2}\left(2.6 \times 10^{2}\right)$ $\mathrm{ng} / \mathrm{L}$, respectively, were approximately $1-2$ orders of magnitude higher than those in the $\mathrm{BC}$ area, 6.9 (5.5) ng/L. Statistically significant differences were observed for OBS levels in samples from these three areas (Mann-Whitney $U$ test, $P<$ 0.05 , as shown in Table 1 ), implying that the oil producing history or oil-well density is an important factor influencing the OBS contamination in the vicinity of petroleum operations. Three samples $(\mathrm{O} 1-\mathrm{O} 3)$ were taken from the same lake near the first oil well dug in 1958 in the Daqing Oilfield. The highest levels of OBS were found at site $\mathrm{O} 2\left(3.2 \times 10^{3} \mathrm{ng} / \mathrm{L}\right), \mathrm{O} 1(2.2$ $\left.\times 10^{3} \mathrm{ng} / \mathrm{L}\right)$ and $\mathrm{O} 3\left(5.8 \times 10^{2} \mathrm{ng} / \mathrm{L}\right)$, further corroborating the conclusion that oil recovery dramatically aggravates OBS contamination in surrounding areas. With the increase of industrialization in China, the oil consumption has been increasing and is beyond the domestic production capacity since $1994 .^{43}$ In addition, the oil production volumes in five of nine giant oilfields, with ultimately recoverable resources more than $7950 \times 10^{4} \mathrm{~m}^{3}$ in China, ${ }^{44}$ have reached the peak value and were expected to decline in the near future. Except those nine giant oilfields, there are a number of smaller or new oilfields, with oil production occupying $30 \%$ of the total amount in China, which will also enter the final stage of oil recovery due to the huge requirements for oil in China. ${ }^{44}$ In view of this situation, various enhanced oil recovery (EOR) techniques (e.g., water flooding, injection of gas or chemical, etc.) were explored to improve the oil production. As effective techniques, some chemicals, especially fluorinated surfactants (e.g., OBS), would be expected to be used more frequently in oilfield of China. In addition, OBS has not only been used to enhance oil recovery but also as an additive in AFFFs and FFFPs in China. ${ }^{27}$ Therefore, we strongly encourage more research to characterize the occurrence of OBS around firefighting training areas. 
Unlike OBS, PFOS was detected at concentrations below 20 $\mathrm{ng} / \mathrm{L}$ in all but four samples, B4 (24 ng/L), O25 (49 ng/L), $\mathrm{O} 20(84 \mathrm{ng} / \mathrm{L})$ and $\mathrm{O} 18\left(1.5 \times 10^{2} \mathrm{ng} / \mathrm{L}\right)$, and the PFOS levels in the selected three areas were not significantly different (Mann-Whitney $U$ test, $P>0.05$ ). Although unverified material has reported that approximately 40-50 tons of PFOS-related compounds were used to enhance oil recovery in 2009 in China, this could not be supported by the observations in the present study from the Daqing Oilfield. The lack of significant correlations (Spearman rank, $P>0.05$ ) observed between PFOS and OBS further indicates that the sources of these two compounds were different. In addition to PFOS, PFBS was detected at concentrations above $10 \mathrm{ng} / \mathrm{L}$ in only five samples (O1-O3, O5, and O6). As mentioned above, $\mathrm{O} 1-\mathrm{O} 3$, in which the highest levels of OBS were detected, are in the same lake near the first oil well. Therefore, the discovery of PFBS may be due to its direct use as an additive in this area or to its presence as an impurity in some related surfactants. The PFCA concentrations in the present study were comparable to those in surface water in China and other countries without obvious point sources ${ }^{45-47}$ and were much lower than those with point sources. ${ }^{36,37}$ Combined the fact that there was no significant difference for PFCAs among the three areas (Mann-Whitney $U$ test, $P>0.05$ ), and no remarkably high levels were detected near the first oil well, PFCAs observed in oilfield area cannot be attributed to any direct use in Daqing Oilfield.

Preliminary Assessment of Toxicity and Environmental Fate. The present study has demonstrated the widespread presence of OBS in the surrounding environment of the Daqing Oilfield, China. Considering that OBS is applied in oil production and firefighting, it is reasonable to suggest that OBS pollution may widely occur in China and other parts of the world. Therefore, its adverse effects on the environment may be of great concern. The acute toxicity of OBS was evaluated using zebrafish and tadpoles as model organisms. During fish acclimatization and frog rearing in the tank, the mortality of zebrafish and tadpoles was $<5 \%$. In the control group, none of the animals died during the acute toxicity test. The deaths and cumulative mortalities of fish and tadpoles in the treatment groups $(11.3,14.5,18.9,24.6$, and $32 \mathrm{mg} / \mathrm{L}$ for zebrafish and 13.7, 19.2, 26.9, 37.6, and $52.7 \mathrm{mg} / \mathrm{L}$ for tadpoles) are shown in SI Table S4. After $96 \mathrm{~h}$, the minimum concentration causing $100 \%$ mortality and the maximum concentration causing no mortality were $32 \mathrm{mg} / \mathrm{L}$ and 18.9 $\mathrm{mg} / \mathrm{L}$ for zebrafish, with $30 \%$ mortality in the $24.6 \mathrm{mg} / \mathrm{L}$ group. As for tadpoles, the minimum concentration causing $100 \%$ mortality and the maximum concentration causing no mortality were $37.6 \mathrm{mg} / \mathrm{L}$ and $19.2 \mathrm{mg} / \mathrm{L}$, with $30 \%$ mortality in the 26.9 $\mathrm{mg} / \mathrm{L}$ group. The LC50 values of OBS for zebrafish and tadpoles were $25.5 \mathrm{mg} / \mathrm{L}$ and $28.4 \mathrm{mg} / \mathrm{L}$, respectively. The $\mathrm{LC}_{50}(96 \mathrm{~h})$ for zebrafish and tadpoles were less than $100 \mathrm{mg} / \mathrm{L}$ but greater than $10 \mathrm{mg} / \mathrm{L}$, which means that OBS should be classified as a Category III chemical in terms of acute toxicity (harmful to aquatic life), according to the Globally Harmonized System (GHS) criteria for the classification of chemicals. ${ }^{48}$ Wang et al. assessed the acute toxicity of PFOS and its alternative F-53B using a similar acute toxicity test. ${ }^{49}$ The $\mathrm{LC}_{50}$ (96 h) values of PFOS and F-53B for zebrafish were $17 \mathrm{mg} / \mathrm{L}$ and $15.5 \mathrm{mg} / \mathrm{L}$, respectively. ${ }^{49}$ Thus, OBS appears to have similar acute toxicity as PFOS according the GHS definition. It should be noted that the concentrations of OBS used in these standardized toxicity tests were several orders of magnitude higher than the environmental levels in Daqing oilfield. Further studies to investigate sublethal chronic effects at lower concentrations are therefore encouraged.

When no acceptable measurements of environmental fate properties are available for chemicals, a screening-level tool, EPI Suite, ${ }^{50}$ can be used to estimate their physicochemical properties, such as the $\log K_{\mathrm{ow}}$, BAF, and persistence, based on different estimation programs. ${ }^{51,52}$ However, for PFASs, this tool can only be used for estimating their environmental degradation half-lives, while the estimation for other physicochemical properties is inaccurate. ${ }^{53}$ Consequently, EPI Suite software was only used to estimate the degradation halflives for OBS in air, water and soil and for PFOS as comparison (detail shown in SI Table S5). Similar half-lives observed for OBS and PFOS mean that OBS would be as persistent as PFOS in the environment after release. These model predictions are also supported by the study of Bao et al. demonstrating that OBS was not readily degraded in closed BOD bottles. ${ }^{26}$ The hydrophobicity of organic compounds plays an important role for determining their transport and fate in the environment. ${ }^{54}$ Based on the relative retention times of OBS $(5.23 \mathrm{~min})$ and PFOS (4.49 $\mathrm{min}$ ) on a reversed-phase C18 column, OBS would be expected to be more hydrophobic than PFOS. Future studies to characterize environmental contamination of OBS should, therefore, include measurements in soils and sediments since this compound will be less efficiently transported with water. As many studies have reported that the bioaccumulation of PFASs is positively related with the hydrophobicity, ${ }^{3}$ it may further be hypothesized that OBS is more bioaccumulative than PFOS. However, further studies are needed to determine how the bulkier structure compared to PFOS affects the uptake, distribution and elimination. Therefore, the bioaccumulation of OBS in aquatic animals in the environment is worth studying further.

Implications. An increasing number of emerging PFASs, with similar persistence and/or bioaccumulation potential to legacy PFASs, ${ }^{23,55}$ have recently been reported to be ubiquitous in environmental and biota samples. ${ }^{56-58}$ In the present study, OBS, a little studied PFAS, was observed with high DF and at higher concentrations compared to PFOS in surface water from an oilfield area, implying an exposure risk to the local environment and its inhabitants. Although the environmental behavior and toxicological effects are currently not clearly known, some compounds containing benzene in their structure have been deemed to be more toxic than their aliphatic counterparts. ${ }^{59,60}$ Through our preliminary toxicity assessment, OBS was classified in Category III according the GHS definition, similar to PFOS. We also preliminarily evaluated its degradation potential via model estimation and found that the estimated degradation time of OBS was approximately 400 days indicating that OBS is a highly recalcitrant pollutant. Considering that most oilfields in China have entered into the final stage of oil production ${ }^{44}$ and that PFOS-based compounds have been restricted or banned globally due to their high persistence and toxicity, OBS is expected to obtain a larger market share in oilfields and other industries where PFOS was once used. In addition, OBS is also used as an additive in AFFF or FFFP, which may result in its widespread releases across China. Therefore, more stringent monitoring of OBS will help evaluate the associated risks of the entire PFAS class and help to close the mass balance of organofluorine compounds environmental samples. 


\section{ASSOCIATED CONTENT}

\section{S Supporting Information}

The Supporting Information is available free of charge on the ACS Publications website at DOI: 10.1021/acs.est.7b04332.

Additional information regarding quality assurance, quality control and other materials are shown in Table S1-S5 and Figure S1-S4 (PDF)

\section{AUTHOR INFORMATION}

\section{Corresponding Author}

*Phonel: +86 (10) 62849239; fax: +86 (10) 62849182; e-mail: caiyaqi@rcees.ac.cn.

ORCID $\odot$

Lin $\mathrm{Xu}$ : 0000-0002-4681-6457

Zhanfen Qin: 0000-0002-3961-0389

Dong Cao: 0000-0001-8793-4401

Yaqi Cai: 0000-0002-2805-5535

\section{Notes}

The authors declare no competing financial interest.

\section{ACKNOWLEDGMENTS}

This work was jointly supported by the National Natural Science Foundation of China (No. 21537004, 21722705, 21677154, 21777182, 21407159) and the Strategic Priority Research Program of the Chinese Academy of Sciences (XDB14010201). We appreciate the constructive comments and editing of the final manuscript by Dr. Robin Vestergren from the Swedish Environmental Research Institute (IVL).

\section{REFERENCES}

(1) Lindstrom, A. B.; Strynar, M. J.; Libelo, E. L. Polyfluorinated compounds: past, present, and future. Environ. Sci. Technol. 2011, 45 (19), 7954-7961.

(2) Parsons, J. R.; Sáez, M.; Dolfing, J.; de Voogt, P. Biodegradation of perfluorinated compounds. Rev. Environ. Contam. Toxicol. 2008, $196,53-71$.

(3) Conder, J. M.; Hoke, R. A.; Wolf, W. d.; Russell, M. H.; Buck, R. C. Are PFCAs bioaccumulative? A critical review and comparison with regulatory criteria and persistent lipophilic compounds. Environ. Sci. Technol. 2008, 42 (4), 995-1003.

(4) UNEP-POPS-COP.4-SC-4-17. Stockholm Convention/WHO International programme on chemical safety Middle East workshop to strengthen capacity for chemicals assessment, 2009, Amman, Jordan.

(5) European Chemical Agency, Candidate list of substances of very high concern for authorisation. 2013, http://echa.europa.eu/web/ guest/candidate-list-table.

(6) Programme, U. N. E., The Stockholm Convention National Implementation Plans Addressing COP 4 amendments. 2016, http:// chm.pops.int/Implementation/NationalImplementationPlans/ NIPTransmission/tabid/253/Default.aspx.

(7) Eriksson, U.; Mueller, J. F.; Toms, L. M. L.; Hobson, P.; Kärrman, A. Temporal trends of PFSAs, PFCAs and selected precursors in Australian serum from 2002 to 2013. Environ. Pollut. 2017, 220 (Part A), 168-177.

(8) Olsen, G. W.; Lange, C. C.; Ellefson, M. E.; Mair, D. C.; Church, T. R.; Goldberg, C. L.; Herron, R. M.; Medhdizadehkashi, Z.; Nobiletti, J. B.; Rios, J. A.; Reagen, W. K.; Zobel, L. R. Temporal trends of perfluoroalkyl concentrations in American Red Cross adult blood donors, 2000-2010. Environ. Sci. Technol. 2012, 46 (11), 63306338.

(9) Glynn, A.; Berger, U.; Bignert, A.; Ullah, S.; Aune, M.; Lignell, S.; Darnerud, P. O. Perfluorinated alkyl acids in blood serum from primiparous women in Sweden: serial sampling during pregnancy and nursing, and temporal trends 1996-2010. Environ. Sci. Technol. 2012, 46 (16), 9071-9079.

(10) Sundström, M.; Ehresman, D. J.; Bignert, A.; Butenhoff, J. L.; Olsen, G. W.; Chang, S. C.; Bergman, A. A temporal trend study (1972-2008) of perfluorooctanesulfonate, perfluorohexanesulfonate, and perfluorooctanoate in pooled human milk samples from Stockholm, Sweden. Environ. Int. 2011, 37 (1), 178-183.

(11) Swedish Chemicals Agency (KEMI), Occurrence and use of highly fluorinated substances and alternatives. 2015, https://www. kemi.se/global/rapporter/2015/report-7-15-occurrence-and-use-ofhighly-fluorinated-substances-and-alternatives.pdf.

(12) Wang, Z.; DeWitt, J. C.; Higgins, C. P.; Cousins, I. T. A neverending story of per- and polyfluoroalkyl substances. Environ. Sci. Technol. 2017, 51 (5), 2508-2518.

(13) Yeung, L. W. Y.; Miyake, Y.; Taniyasu, S.; Wang, Y.; Yu, H. X.; So, M. K.; Jiang, G. B.; Wu, Y. N.; Li, J. G.; Giesy, J. P.; Yamashita, N.; Lam, P. K. S. Perfluorinated compounds and total and extractable organic fluorine in human blood samples from China. Environ. Sci. Technol. 2008, 42 (21), 8140-8145.

(14) Yeung, L. W. Y.; Mabury, S. A. Are humans exposed to increasing amounts of unidentified organofluorine? Environ. Chem. 2016, 13 (1), 102.

(15) Schymanski, E. L.; Williams, A. J. Open Science for identifying "Known Unknown" Chemicals. Environ. Sci. Technol. 2017, 51, 53575359.

(16) Strynar, M.; Dagnino, S.; McMahen, R.; Liang, S.; Lindstrom, A.; Andersen, E.; McMillan, L.; Thurman, M.; Ferrer, I.; Ball, C. Identification of novel perfluoroalkyl ether carboxylic acids (PFECAs) and sulfonic acids (PFESAs) in natural waters using accurate mass time-of-flight mass spectrometry (TOFMS). Environ. Sci. Technol. 2015, 49 (19), 11622-11630.

(17) Gebbink, W. A.; Asseldonk, L.; van Leeuwen, S. P. J. Presence of emerging per- and polyfluoroalkyl substances (PFASs) in river and drinking water near a fluorochemical production plant in the Netherlands. Environ. Sci. Technol. 2017, 51, 11057-11065.

(18) Newton, S.; McMahen, R; Stoeckel, J. A.; Chislock, M.; Lindstrom, A.; Strynar, M. Novel polyfluorinated compounds identified using high resolution mass spectrometry downstream of manufacturing facilities near Decatur, Alabama. Environ. Sci. Technol. 2017, 51 (3), 1544-1522.

(19) Place, B. J.; Field, J. A. Identification of novel fluorochemicals in aqueous film-forming foams used by the US military. Environ. Sci. Technol. 2012, 46 (13), 7120-7127.

(20) D'Agostino, L. A.; Mabury, S. A. Identification of novel fluorinated surfactants in aqueous film forming foams and commercial surfactant concentrates. Environ. Sci. Technol. 2014, 48 (1), 121-129.

(21) Barzen-Hanson, K. A.; Roberts, S. C.; Choyke, S.; Oetjen, K.; McAlees, A.; Riddell, N.; McCrindle, R.; Lee Ferguson, P.; Higgins, C. P.; Field, J. A. Discovery of 40 classes of per- and polyfluoroalkyl substances in historical aqueous film-forming foams (AFFFs) and AFFF-impacted groundwater. Environ. Sci. Technol. 2017, 51, 20472057.

(22) Liu, Y.; Pereira, A. D. S.; Martin, J. M. Discovery of C5-C17 poly- and perfluoroalkyl substances in water by In-line SPE-HPLCOrbitrap with In-source fragmentation flagging. Anal. Chem. 2015, 87, 4260-4268.

(23) Shi, Y.; Vestergren, R.; Xu, L.; Zhou, Z.; Li, C.; Liang, Y.; Cai, Y. Human exposure and elimination kinetics of chlorinated polyfluoroalkyl ether sulfonic acids (Cl-PFESAs). Environ. Sci. Technol. 2016, 50 (5), 2396-2404.

(24) Wang, Y.; Vestergren, R.; Shi, Y.; Cao, D.; Xu, L.; Cai, Y.; Zhao, $\mathrm{X}$.; Wu, F. Identification, tissue distribution, and bioaccumulation potential of cyclic perfluorinated sulfonic acids isomers in an airport impacted ecosystem. Environ. Sci. Technol. 2016, 50 (20), 1092310932.

(25) Funasaki, N., Hada, S., Mutual solubility in mixed micelles of fluorocarbon and hydrocarbon surfactants from surface tension data, Chemistry Letters, 1979, 717-718. 
(26) Bao, Y.; Qu, Y.; Huang, J.; Yu, G. First assessment on degradability of sodium p-perfluorous nonenoxybenzene sulfonate (OBS), a high volume alternative to perfluorooctane sulfonate in firefighting foams and oil production agents in China. RSC Adv. 2017, 7, 46948-46957.

(27) Chen, Z. New fluorinated surfactant-OBS. Shanghai Chemical Industry (Chinese) 1984, 9 (2), 33-35.

(28) Liao, K.; Ge, J.; Zhang, G.; Fu, L.; He, J.; Ren, C. A kind of fluorine-containing cleanup agent for fracturing in oilfield (Chinese). 2016, Patent Number: CN 105694840A.

(29) Sha, M.; Xing, P.; Jiang, B. Strategies for synthesizing nonbioaccumulable alternatives to PFOA and PFOS. Chin. Chem. Lett. 2015, 26, 491-498.

(30) Chen, L.; Shi, H.; Wu, H.; Xiang, J. Synthesis and combined properties of novel fluorinated anionic surfactant. Colloids Surf., A 2011, 384, 331-336.

(31) Cao, Y.; Li, F.; Zhang, Y. Synthesis, characterization and application of sodium $p$-perfluorous nonenoxybenzene sulfonate. Fine Chemicals (in Chinese) 2012, 29 (2), 174-177.

(32) Ghoulam, M. B.; Moatadid, N.; Graciaa, A.; Marion, G.; Lachaise, J. Hydrocarbon/Fluorocarbon mixed micelle diagram from surface tensiometry. Langmuir 1996, 12, 5048-5051.

(33) Graciaa, A.; Creux, P.; Lachaise, J.; Schechter, R. S. Competitive adsorption of surfactants at air/water interfaces. J. Colloid Interface Sci. 2003, 261, 233-237.

(34) Oil industry released tons of toxic pollutant. 2005, http://www. fluoridealert.org/wp-content/pesticides/pfoa.pfos.intro.html.

(35) Moody, C. A.; Field, J. A. Perfluorinated surfactants and the environmental implications of their use in fire-fighting foams. Environ. Sci. Technol. 2000, 34 (18), 3864-3870.

(36) Zhou, Z.; Liang, Y.; Shi, Y.; Xu, L.; Cai, Y. Occurrence and transport of perfluoroalkyl acids (PFAAs), including short-chain PFAAs in Tangxun Lake, China. Environ. Sci. Technol. 2013, 47 (16), 9249-9257.

(37) Shi, Y.; Vestergren, R.; Xu, L.; Song, X.; Niu, X.; Zhang, C.; Cai, Y. Characterizing direct emissions of perfluoroalkyl substances from ongoing fluoropolymer production sources: A spatial trend study of Xiaoqing River, China. Environ. Pollut. 2015, 206, 104-112.

(38) Organisation for Economic Cooperation and Development (OECD) (301D) Guideline for Testing of Chemicals (closed bottle test), 1992.

(39) Su, H.; Ren, D.; Cao, S.; Qin, Z. Acute toxicity of perfluorooctane (PFOS) and its substitutes to amphibian tadpoles. Asian J. Ecotoxicol. 2012, 7, 521-524.

(40) Lou, Q. C. S.; Xu, W.; Zhang, Y.; Qin, Z.; Wei, W. Molecular characterization and mRNA expression of ribosomal protein L8 in Rana nigromaculata during development and under exposure to hormones. J. Environ. Sci. 2014, 26 (11), 2331-2339.

(41) Gosner, K. L. A simplified table for staging anuran embryos and larvae withnotes on identification. Herpetologica 1960, 16, 183-190.

(42) Schymanski, E. L.; Jeon, J.; Gulde, R.; Fenner, K.; Ruff, M.; Singer, H. P.; Hollender, J. Identifying small molecules via high resolution mass spectrometry: Communicating confidence. Environ. Sci. Technol. 2014, 48, 2097-2098.

(43) Musa, S. D.; Tang, Z.; Ibrahim, A. O.; Habib, M. China’s energy status: A critical look at fossils and renewable options. Renewable Sustainable Energy Rev. 2017, .81228110.1016/j.rser.2017.06.036

(44) Mikael, H.; Tang, X.; Rang, X.; Kjell, A. Development journey and outlook of Chinese giant oilfields. Petrol. Explor. Develop. 2010, 37 (2), 237-249.

(45) Cai, M.; Zhao, Z.; Yang, H.; Yin, Z.; Hong, Q.; Sturm, R.; Ebinghaus, R.; Ahrens, L.; Cai, M.; He, J.; Xie, Z. Spatial distribution of per- and polyfluoroalkyl compounds in coastal waters from the East to South China Sea. Environ. Pollut. 2012, 161, 162-169.

(46) Shao, M.; Ding, G.; Zhang, J.; Wei, L.; Xue, H.; Zhang, N.; Li, Y.; Chen, G.; Sun, Y. Occurrence and distribution of perfluoroalkyl substances (PFASs) in surface water and bottom water of the Shuangtaizi Estuary, China. Environ. Pollut. 2016, 216, 675-681.
(47) Wang, T.; Khim, J. S.; Chen, C.; Naile, J. E.; Lu, Y.; Kannan, K.; Park, J.; Luo, W.; Jiao, W.; Hu, W.; Giesy, J. P. Perfluorinated compounds in surface waters from Northern China: comparison to level of industrialization. Environ. Int. 2012, 42, 37-46.

(48) United Nations. Globally Harmonized System of Classification and Labelling of Chemicals (GHS), ST/SG/AC.10/30/Rev.4. 2011.

(49) Wang, S.; Huang, J.; Yang, Y.; Hui, Y.; Ge, Y.; Larssen, T.; Yu, G.; Deng, S.; Wang, B.; Harman, C. First report of a Chinese PFOS alternative overlooked for 30 years: its toxicity, persistence, and presence in the environment. Environ. Sci. Technol. 2013, 47 (18), $10163-10170$.

(50) U.S. EPA. Exposure Assessment Tools and Models, Estimation Program Interface (EPI) Suite, V 4.11; U.S. Environmental Protection Agency, Exposure Assessment Branch: Washington, DC, 2012.

(51) Strempel, S.; Scheringer, M.; Ng, C. A.; Hungerbuhler, K. Screening for PBT chemicals among the "existing" and "new" chemicals of the EU. Environ. Sci. Technol. 2012, 46 (11), 5680-5687.

(52) Zarfl, C.; Hotopp, I.; Kehrein, N.; Matthies, M. Identification of substances with potential for long range transport as possible substances of very high concern. Environ. Sci. Pollut. Res. 2012, 19 (8), 3152-3161.

(53) Arp, H. P. H.; Niederer, C.; Goss, K. U. Predicting the partitioning behavior of various highly fluorinated compounds. Environ. Sci. Technol. 2006, 40 (23), 7298-304.

(54) Vailaya, A.; Horvath, C. Retention in reversed-phase chromatography: partition or adsorption. J. Chromat. A 1998, 829, $1-2$.

(55) Shi, Y.; Vestergren, R.; Zhou, Z.; Song, X.; Xu, L.; Liang, Y.; Cai, Y. Tissue distribution and whole body burden of the chlorinated polyfluoroalkyl ether sulfonic acid F-53B in Crucian Carp (Carassius carassius): Evidence for a highly bioaccumulative contaminant of emerging concern. Environ. Sci. Technol. 2015, 49, 14156-14165.

(56) Ruan, T.; Lin, Y.; Wang, T.; Liu, R.; Jiang, G. Identification of novel polyfluorinated ether sulfonates as PFOS alternatives in municipal sewage sludge in China. Environ. Sci. Technol. 2015, 49 (11), 6519-6527.

(57) Chu, S.; Letcher, R. J.; McGoldrick, D. J.; Backus, S. M. A new fluorinated surfactant contaminant in biota: perfluorobutane sulfonamide in several fish species. Environ. Sci. Technol. 2016, 50 (2), 669675.

(58) Gebbink, W. A.; Bossi, R.; Riget, F. F.; Rosing-Asvid, A.; Sonne, C.; Dietz, R. Observation of emerging per- and polyfluoroalkyl substances (PFASs) in Greenland marine mammals. Chemosphere 2016, 144, 2384-2391.

(59) He, J.; Li, J.; Wen, Y.; Tai, H.; Yu, Y.; Qin, W.; Su, L.; Zhao, Y. Investigation on modes of toxic action to rats based on aliphatic and aromatic compounds and comparison with fish toxicity based on exposure routes. Chemosphere 2015, 128, 111-117.

(60) Liu, L.; Zhang, X. Comparative toxicity of new halophenolic DBPs in chlorinated saline wastewater effluents against a marine alga: Halophenolic DBPs are generally more toxic than haloaliphatic ones. Water Res. 2014, 65, 64-72. 